\title{
Comparison of circulating lipid profiles between fasting humans and three animal species used in preclinical studies: mice, rats and rabbits
}

\author{
Masaki Ishikawa', Kosuke Saito ${ }^{1 *}$, Masayo Urata', Yuji Kumagai ${ }^{2}$, Keiko Maekawa ${ }^{1}$ and Yoshiro Saito ${ }^{1}$
}

\begin{abstract}
Background: Circulating lipid metabolites are associated with many physiological and biological processes in the body, and therefore could be used as biomarkers for evaluating drug efficacy and safety in preclinical studies. However, differences in circulating lipid profiles among humans and animals often used in preclinical studies have not been fully investigated.
\end{abstract}

Methods: We performed lipidomic analysis to obtain circulating lipid profiles of fasted humans (Caucasian, $n=15)$ and three animal species used in preclinical studies (mice [BALB/C, $n=5$ ], rats [Sprague-Dawley, $n=5$ ], and rabbits [New Zealand White, $n=5]$ ]) by using liquid chromatography-mass spectrometry.

Results: Our data showed marked differences in lipid profiles among humans and these animal species. Furthermore, we observed that the levels of many lipid metabolites, such as poly-unsaturated fatty acid-containing cholesteryl esters, ether-type phosphoglycerolipids, and sulfatides, were significantly different $(p<0.05)$ by more than 10 -fold in these animals (depending on the animal species) from humans.

Conclusion: Our data could be useful while extrapolating the data on the biomarker candidates identified in preclinical studies into clinical studies.

Keywords: Preclinical studies, LC-MS, Circulating lipid metabolites, Lipidomic analysis

\section{Background}

Lipid metabolites, such as phosphoglycerolipids (PLs) and sphingolipids (SLs), are components of the cell membrane, and play key roles in a variety of physiological and biological processes including apoptosis [1] and autophagy [2]. These lipid metabolites are also the major components of plasma lipoproteins, including low- and high-density lipoproteins. Because somatic tissues uptake and/or excrete lipid metabolites through lipoproteins, the levels of circulating lipid metabolites are closely related to the levels of lipid metabolites in tissues [3]. Thus, circulating lipid metabolites might be associated with many physiological and biological processes in the whole body, and therefore might help in selecting useful biomarkers for evaluating diseased states and drug responses.

\footnotetext{
* Correspondence: saitok2@nihs.go.jp

'Division of Medicinal Safety Science, National Institute of Health Sciences,

1-18-1 Kamiyoga, Setagaya, Tokyo 158-8501, Japan

Full list of author information is available at the end of the article
}

Lipidomics is a metabolomics approach focused on lipid metabolites $[4,5]$ that can overview the lipid profile of biofluids and tissues, and hence is used as a highthroughput tool for simultaneously identifying various biomarker candidates. So far, lipidomics has already been applied not only in clinical studies [6-8], but also in preclinical pharmacology and toxicology studies [9-13]. This is because circulating lipid metabolites are common among animals used in preclinical studies and humans, and thus can be used to simplify the extrapolation of data on biomarkers identified in preclinical studies into clinical studies. However, differences in circulating lipid profiles among humans and animals used in preclinical studies have not been fully investigated.

In the present study, we employed lipidomic analysis to obtain circulating lipid profiles of humans (Caucasian, $n=15)$ and three animal species used in preclinical studies (mice $[\mathrm{BALB} / \mathrm{c}, n=5]$, rats [Sprague-Dawley, $n=5$ ], and rabbits [New Zealand White, $n=5$ ]) by using liquid 
chromatography-mass spectrometry (LC-MS). To minimize gender and age-associated variation affecting the differences in lipid metabolites, we selected young adult males which are generally used in the preclinical and phase I studies. We observed marked differences in lipid profiles between humans and all the selected animal species. In addition, our data showed that the levels of many lipid metabolites, such as poly-unsaturated fatty acid (PUFA)-containing cholesteryl esters (ChEs), ether-type PLs and sulfatides (Suls), are more than 10-fold higher in the selected animals (depending on the animal species) than in humans. These findings would provide useful fundamental information for selecting biomarker candidates obtained/identified in preclinical studies for future extrapolation into clinical studies.

\section{Material and methods}

\section{Human plasma}

Blood samples from healthy white males were purchased from PromedDX (Norton, MA). The ethics committee of the National Institute of Health Sciences authorized PromedDX as a validated provider of blood samples and exempted us from the committee's approval for use of the purchased blood samples. The samples were collected after obtaining written informed consent from all human subjects; the subjects were 15 young men (25-33-years-old) who had been fasting for more than $14 \mathrm{~h}$. Fresh blood from each individual was collected and simultaneously drawn into $10 \mathrm{ml}$ Vacutainer Plasma Separator Tubes containing EDTA (Becton Dickinson, Franklin Lakes, NJ), which is needed for plasma separation. Plasma was separated within $2 \mathrm{~h}$ after the collection of blood samples. The plasma samples were immediately frozen and stored at $-80{ }^{\circ} \mathrm{C}$. After shipment with dry ice from PromedDX, all frozen samples were thawed once on ice and divided into small aliquots before storing at $-80{ }^{\circ} \mathrm{C}$ until lipid extraction.

\section{Animal plasma}

Plasma samples of male BALB/c mice ( $n=5,10$-weeks-old) and male New Zealand white rabbits $(n=5,10$-weeks-old) were purchased from KOHJIN BIO (Saitama, Japan). The plasma sample of male Sprague-Dawley rats $(n=5$, 8-weeks-old; Charles River Japan, Kanagawa, Japan) was prepared in our laboratory. All plasma samples were separated within $2 \mathrm{~h}$ after collection of blood samples by using EDTA as an anticoagulant. The plasma samples were obtained from all animals after $16 \mathrm{~h}$ of fasting. The plasma samples were immediately frozen and stored at $-80{ }^{\circ} \mathrm{C}$. The experimental protocols for the animal procedures were approved by the Ethics Review Committee for Animal Experimentation of the National Institute of Health Sciences (Tokyo, Japan).

\section{Lipid extraction}

Lipid extraction and measurement of lipid metabolites by LC-MS was performed as reported previously $[14,15]$. In brief, lipid metabolites were extracted from $90 \mu \mathrm{L}$ of plasma for humans and animal species, by using the Bligh and Dyer method with a few modifications. Briefly, plasma was mixed with $3.71 \mathrm{~mL}$ of chloroform/methanol/20 mM Kpi (1:2:0.8) for 5 min. 12:0-12:0 phosphatidylethanolamine (12:0-12:0 PE, Avanti, Polar Lipids, Inc., Alabaster, $\mathrm{AL}$ ) was used as the internal standard (IS). Next, $1 \mathrm{~mL}$ each of chloroform and $20 \mathrm{mM}$ Kpi was added, mixed for $5 \mathrm{~min}$, and centrifuged at $1000 \times \mathrm{g}$ for $10 \mathrm{~min}$. After discarding upper layers, $3.2 \mathrm{~mL}$ of chloroform/methanol/ $100 \mathrm{mM} \mathrm{KCl} \mathrm{(3:47:48)}$ were added, mixed, and then centrifuged at $1000 \times \mathrm{g}$ for $10 \mathrm{~min}$ to separate the lower organic layer. The lower organic layers were supplied to non-targeted lipidomic analysis.

\section{Non-targeted lipidomics}

The lower organic layers were used for the measurement of PLs, SLs, and neutral lipids (NLs) using LC-time of flight (TOF)-MS (Acquity UPLC system-LCT Premier XE; Waters, Milford, MA). The samples from each group were randomized across the LC-TOF-MS measurement. PLs and SLs were detected in the negative ion mode, while NLs were detected in the positive ion mode. Data obtained from LC-TOF-MS were processed using the 2DICAL software (Mitsui Knowledge Industry, Tokyo, Japan). The extracted ion peaks were analyzed

Table 1 Circulating lipid metabolites identified by non-targeted lipidomic analysis of each mammal

\begin{tabular}{llll}
\hline Lipid types & Ion mode & Lipid classes & Number \\
\hline Phosphoglycerolipids & Negative & lysoPC & 11 \\
& & lysoPE & 5 \\
& PC & 45 \\
& ePC & 19 \\
& PE & 13 \\
& ePE & 18 \\
Sphingolipids & PI & 11 \\
& & SM & 26 \\
& Cer & 5 \\
Neutral lipids & HexCer & 5 \\
& & Sul & 3 \\
& Positive & DG & 12 \\
& & Ch/ChE & 31 \\
& CoQ & 2 \\
& Total & 206 \\
\hline
\end{tabular}

lysoPC lysophosphatidylcholine, lysoPE lysophosphatidylethanolamine, $P C$ phosphatidylcholine, ePC ether-type phosphatidylcholine, $P E$ phosphatidylethanolamine, ePE ether-type phosphatidylethanolamine, PI phosphatidylinositol, SM sphingomyelin, Cer ceramide, $\mathrm{Hex} C e r$ hexosylceramide, DG diacylglycerol, Ch/ChE cholesterol/cholesteryl ester, CoQ coenzyme Q 


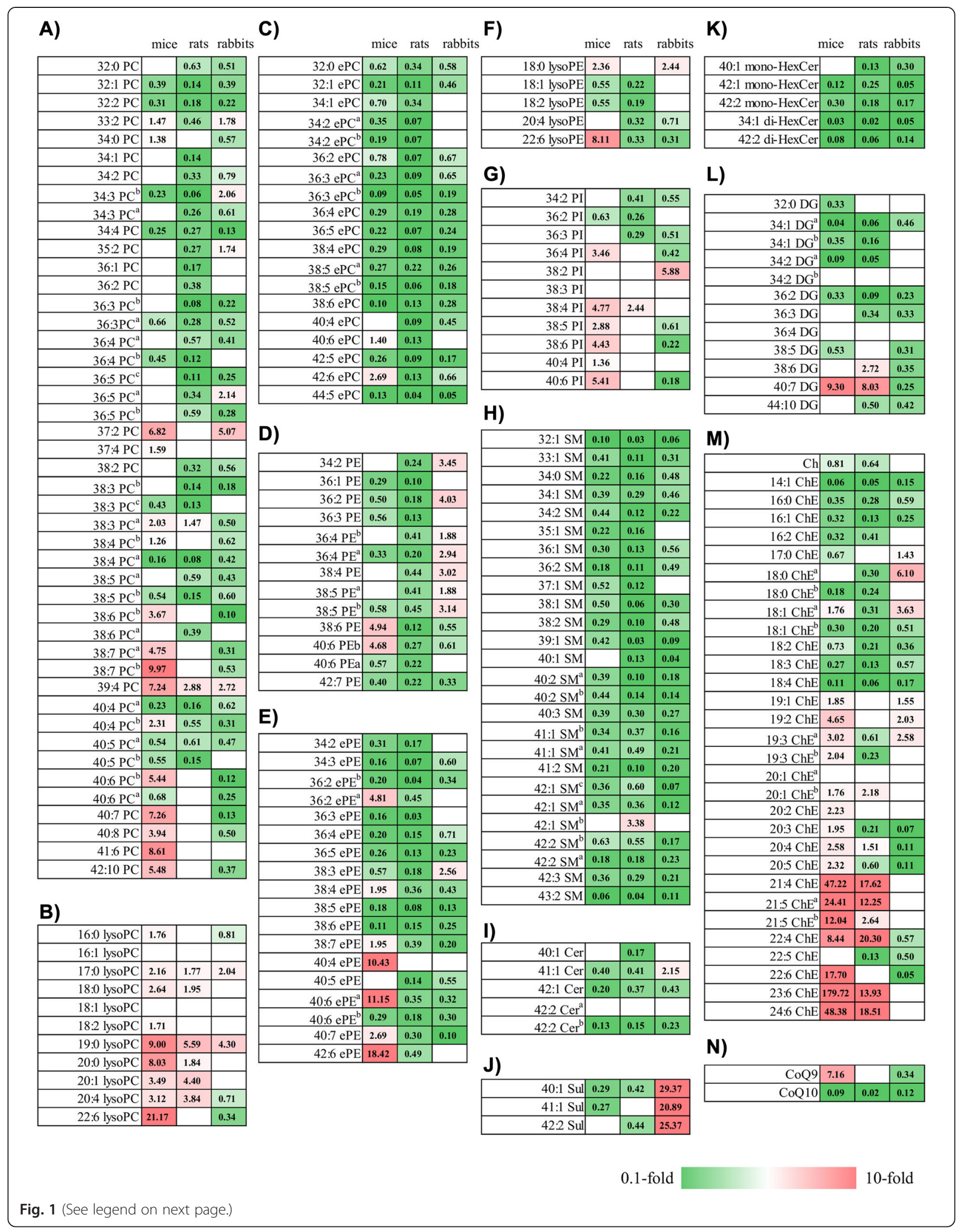


(See figure on previous page.)

Fig. 1 Heat maps of lipid metabolites among humans and three animal species. The heat map with significantly different ( $p<0.05)$ lipid metabolites were generated using mean fold changes in the levels of metabolites calculated as ratios of each animal species to human. Vacant white cells indicate metabolites that were detected, but their levels were not significantly different. (a) PC, (b) lysoPC, (c) ePC, (d) PE, (e) ePE, (f) lysoPE, (g) PI, (h) SM, (i) Cer, (k) HexCer, (I) DG, (m) Ch/ChE and (n) CoQ. If more than two lipid species possessed the same chemical formula, alphabetical letters were put after each lipid molecule class to distinguish them from each other (Additional file 1: Table S1). PC, phosphatidylcholine; lysoPC, lysophosphatidylcholine; ePC, ether-type phosphatidylcholine; PE, phosphatidylethanolamine; lysoPE, lysophosphatidylethanolamine; ePE; ether-type phosphatidylethanolamine; PI, phosphatidylinositol; SM, sphingomyelin; Cer, ceramide; HexCer, hexosylceramide; DG, diacylglycerol; Ch/ChE, cholesterol/cholesteryl ester; CoQ, coenzyme Q

for identifying lipid metabolites by comparison of the ion features including $\mathrm{RT}, \mathrm{m} / \mathrm{z}$, preferred adducts, and in-source fragments of the experimental samples with those of our reference library of lipid metabolite entries. The levels of the identified lipid metabolites were normalized to those of IS. If more than two lipid species possessed the same chemical formula, alphabetical letters were put after each lipid molecule class to distinguish them from each other.

\section{Statistical analysis}

The statistical analyses were performed using Welch's $t$-test with Bonferroni correction for the comparison of metabolite levels among humans and different animal species. Differences with $p$ values less than 0.05 were considered statistically significant.

\section{Results and discussion}

Lipidomic analysis of human and preclinical animal plasma To investigate differences in circulating lipid profiles among humans and three animals often used in preclinical studies, we performed a non-targeted lipidomic analysis using plasma samples obtained from each mammal. To maintain experimental quality throughout extraction and measurement, the coefficient of variation for the IS was calculated as $11.7 \%$. In total, we identified 206 lipid metabolites consisting of 122 PLs, 39 SLs, and 45 NLs (Table 1 and Additional file 1: Table S1). Of the 206 identified lipid metabolites in all the mammals, the levels of 163,166 , and 151 metabolites were significantly different in mice, rats, and rabbits, respectively, when compared with the levels of those metabolites in humans (Fig. 1).

As shown in Fig. 1a, the levels of many phosphatidylcholines (PCs) were significantly lower in the three animals than in humans, although relatively longer and highly unsaturated PCs showed significantly higher levels in mice than in humans. The levels of many phosphatidylethanolamines (PEs) were significantly lower in rodents than in humans, while those of many PEs were significantly higher in rabbits than in humans (Fig. 1d). Among ether-type PCs and PEs (ePCs and ePEs), many metabolites showed significantly lower levels in the three selected animal models than in humans, with several exceptions in mice (Fig. 1c and e). Phosphatidylinositols (PIs) showed significantly higher levels in mice than in humans (Fig. 1g). In contrast, the levels of many PIs were significantly lower in rabbits than in humans. As shown in Fig. 1m, the levels of many ChEs were significantly different between humans and the selected animal species, although the differences varied in the three animal species.

Recently, lysoPCs and SLs have been proposed as biomarker candidates for the prediction and evaluation of diseased states and drug safety, respectively, such acetaminophen-induced liver injury [9], drug-induced phospholipidosis and $\beta$-cell dysfunction $[12,16]$ in preclinical studies. In the present study, most of the 11 lysoPCs (9 in mice and 6 in rats) were present at significantly higher levels in rodents than in humans (Fig. 1b). On the other hand, most of the 39 SLs (33 in mice, 36 in rats and 30 in rabbits) were present at significantly lower levels in all the three animal species which are often used in preclinical studies than in humans, although Suls showed significant higher levels in rabbits than in other mammals (Fig. 1h-k). These results indicated that broad molecules of lysoPCs and SLs show different levels in human and animal species. Therefore, species differences should be carefully considered when these lipid molecules are selected as biomarker candidates identified in preclinical studies for extrapolation and usage in clinical studies.

\section{Lipid metabolites showing more than 10 -fold difference in their levels between humans and animal species}

The extent of differences in the levels of lipid metabolites between humans and animal species that are used in preclinical studies seems to be the key problem for the extrapolation of biomarker candidates identified in preclinical studies into clinical studies. Because the range of fold change for the reported lipid biomarkers identified using animals species were approximately $0.5-8.0$-fold $[9,12,13,16]$, the lipid metabolites that exceeded this range might be unsuitable as biomarker candidates in humans. We therefore focused on the lipid metabolites that showed greater than 10 -fold value and significantly different levels in animal species as compared to their levels in humans (Tables 2, 3, 4). The numbers of these lipid metabolites were 19, 37, and 12 for mice, rats, and rabbits, respectively. 
Table 2 Lipid metabolites that showed more than 10-fold and significant differences between humans and mice

\begin{tabular}{|c|c|c|c|}
\hline \multicolumn{2}{|l|}{$>10$-fold } & \multicolumn{2}{|l|}{$<10$-fold } \\
\hline Metabolite & Fold & Metabolite & Fold \\
\hline 22:6 lysoPC & 21.1692 & $36: 3 \mathrm{ePC}^{\mathrm{b}}$ & 0.0887 \\
\hline $21: 4 \mathrm{ChE}$ & 47.2233 & $38: 6 \mathrm{ePC}$ & 0.0973 \\
\hline 21:5 $\mathrm{ChE}^{\mathrm{a}}$ & 24.4064 & $43: 2$ SM & 0.0648 \\
\hline 21:5 $\mathrm{ChE}^{\mathrm{b}}$ & 12.0447 & 34:1 di-HexCer & 0.0293 \\
\hline 22:6 ChE & 17.7039 & 42:2 di-HexCer & 0.0790 \\
\hline 23:6 ChE & 179.7175 & $34: 1 D^{a}$ & 0.0409 \\
\hline 24:6 ChE & 48.3842 & $34: 2 \mathrm{DG}^{\mathrm{a}}$ & 0.0935 \\
\hline 40:4 ePE & 10.4272 & 14:1 ChE & 0.0610 \\
\hline $40: 6 \mathrm{ePE}^{\mathrm{a}}$ & 11.1450 & CoQ10 & 0.0899 \\
\hline 42:6 ePE & 18.4164 & & \\
\hline
\end{tabular}

If more than two lipid species possessed the same chemical formula, alphabetical letters were put after each lipid molecule class to distinguish them from each other (Additional file 1: Table S1)

lysoPC lysophosphatidylcholine, ChE cholesteryl ester, ePE ether-type phospha tidylethanolamine, $e P C$ ether-type phosphatidylcholine, $S M$ sphingomyelin,

HexCer hexosylceramide, DG diacylglycerol, CoQ coenzyme $Q$

Interestingly ChEs with PUFA, such as 23:6 and 24:6 ChE, showed markedly higher levels (179.7175- and 48.3842-fold, respectively) in mice than in humans (Table 2). These large changes of the PUFA-containing ChEs were also consistent with the results obtained in rats, showing 13.9319-fold and 18.5058-fold changes for 23:6 and 24:6 ChE, respectively (Table 3). These results indicated that the levels of circulating PUFA-containing ChEs are higher in rodents than in humans. However, the mechanism underlying the species-specific difference in these levels remains unknown.

As shown in Table 3, 15 ether-type PLs, such as 36:3 $\mathrm{ePC}^{\mathrm{b}}$ and 36:3 ePE, were present in drastically lower levels (less than 0.1-fold) in rats as compared to their levels in humans (the range of fold changes; 0.03310.0947). In addition, all other ether-type PLs, except 40:4 $\mathrm{ePE}$, were significantly lower in rats than in humans (Fig. 1c and e). Moreover, many ether-type PLs showed significantly lower levels in mice than in humans (Fig. 1c and e), although the ether-type PLs which showed less than 0.1 -fold change and significantly lower levels were only found for 36:3 $\mathrm{ePC}^{\mathrm{b}}$ and 38:6 ePC (Table 2). These observations suggested that circulating basal levels of many ether-type PLs are lower in rodents than in humans. The mechanism underlying this difference in the levels of ether-type PLs in rodents remains unknown and needs to be investigated in the future studies.

Rabbits are often used in preclinical studies in addition to rodents. All three Suls showed more than 20-fold higher levels in rabbits than in humans (Table 4). Suls have been proposed as biomarkers for neurodegenerative
Table 3 Lipid metabolites that showed more than 10-fold and significant differences between humans and rats

\begin{tabular}{|c|c|c|c|}
\hline \multicolumn{2}{|l|}{$>10$-fold } & \multicolumn{2}{|l|}{$<10$-fold } \\
\hline Metabolite & Fold & Metabolite & Fold \\
\hline 21:4 ChE & 17.6192 & $34: 3 P C^{b}$ & 0.0648 \\
\hline 21:5 $\mathrm{ChE}^{\mathrm{a}}$ & 12.2477 & $36: 3 P C^{b}$ & 0.0827 \\
\hline 22:4 ChE & 20.3018 & $38: 4 P C^{a}$ & 0.0844 \\
\hline 23:6 ChE & 13.9319 & $34: 2 \mathrm{ePC}^{\mathrm{a}}$ & 0.0717 \\
\hline \multirow[t]{28}{*}{ 24:6 ChE } & 18.5058 & $34: 2 \mathrm{ePC}^{\mathrm{b}}$ & 0.0713 \\
\hline & & $36: 2$ ePC & 0.0724 \\
\hline & & $36: 3 \mathrm{ePC}^{\mathrm{a}}$ & 0.0934 \\
\hline & & $36: 3 \mathrm{ePC}^{\mathrm{b}}$ & 0.0516 \\
\hline & & $36: 5$ ePC & 0.0729 \\
\hline & & $38: 4$ ePC & 0.0782 \\
\hline & & $38: 5 \mathrm{ePC}^{\mathrm{b}}$ & 0.0600 \\
\hline & & 40:4 ePC & 0.0877 \\
\hline & & 42:5 ePC & 0.0947 \\
\hline & & $44: 5 \mathrm{ePC}$ & 0.0411 \\
\hline & & 36:1 PE & 0.0997 \\
\hline & & $34: 3$ ePE & 0.0656 \\
\hline & & $36: 2 e^{P} E^{b}$ & 0.0409 \\
\hline & & $36: 3$ ePE & 0.0331 \\
\hline & & 38:5 ePE & 0.0778 \\
\hline & & $32: 1 \mathrm{SM}$ & 0.0293 \\
\hline & & 38:1 SM & 0.0626 \\
\hline & & 39:1 SM & 0.0339 \\
\hline & & $40: 2 \mathrm{SM}^{a}$ & 0.0957 \\
\hline & & 43:2 SM & 0.0401 \\
\hline & & 34:1 di-HexCer & 0.0186 \\
\hline & & 42:2 di-HexCer & 0.0643 \\
\hline & & $34: 1 \mathrm{DG}^{\mathrm{a}}$ & 0.0648 \\
\hline & & $34: 2 \mathrm{DG}^{\mathrm{a}}$ & 0.0523 \\
\hline & & $36: 2 \mathrm{DG}$ & 0.0945 \\
\hline & & 14:1 ChE & 0.0513 \\
\hline & & 18:4 ChE & 0.0565 \\
\hline & & CoQ10 & 0.0174 \\
\hline
\end{tabular}

If more than two lipid species possessed the same chemical formula, alphabetical letters were put after each lipid molecule class to distinguish them from each other (Additional file 1: Table S1)

ChE cholesteryl ester, $P C$ phosphatidylcholine, $e P C$ ether-type phosphatidylcho line, $P E$ phosphatidylethanolamine, $e P E$ ether-type phosphatidylethanolamine, $S M$ sphingomyelin, HexCer hexosylceramide, DG diacylglycerol, CoQ coenzyme Q

diseases such as Alzheimer's disease [17] and multiple sclerosis [18]. Therefore, when Suls are identified as biomarker candidates for disease states and drug responses in rabbits, their extrapolation into human clinical trials calls for extensive study and needs attention. 
Table 4 Lipid metabolites that showed more than 10-fold and significant differences between humans and rabbits

\begin{tabular}{|c|c|c|c|}
\hline \multicolumn{2}{|l|}{$>10$-fold } & \multicolumn{2}{|l|}{$<10$-fold } \\
\hline Metabolite & Fold & Metabolite & Fold \\
\hline 40:1 Sul & 29.3688 & 44:5 ePC & 0.0541 \\
\hline 41:1 Sul & 20.8891 & $32: 1 \mathrm{SM}$ & 0.0551 \\
\hline \multirow[t]{7}{*}{ 42:2 Sul } & 25.3672 & $39: 1 \mathrm{SM}$ & 0.0858 \\
\hline & & $40: 1 \mathrm{SM}$ & 0.0402 \\
\hline & & $42: 1 \mathrm{SM}^{\mathrm{C}}$ & 0.0701 \\
\hline & & 42:1 mono-HexCer & 0.0465 \\
\hline & & 34:1 di-HeXCer & 0.0475 \\
\hline & & 20:3 ChE & 0.0666 \\
\hline & & 22:6 ChE & 0.0528 \\
\hline
\end{tabular}

If more than two lipid species possessed the same chemical formula, alphabetical letters were put after each lipid molecule class to distinguish them from each other (Additional file 1: Table S1)

Sul sulfatide, ePC ether-type PC, SM sphingomyelin, HexCer, hexosylceramide, ChE cholesteryl ester

\section{Conclusions}

We compared the profiles of circulating lipid metabolites between humans and three animal species often used in preclinical studies by using a non-targeted lipidomics approach. By comparing these profiles, we revealed that some of circulating lipid metabolites were present at markedly different levels between humans and animal species. Our results therefore provide useful and fundamental information for selecting biomarker candidates that might be identified in future preclinical studies for their successful extrapolation into human clinical studies.

\section{Additional file}

Additional file 1: Table S1. Normalized peak height of phosphoglycerolipids, sphingolipids, and neutral lipids in the plasma of all the mammals. (XLSX $112 \mathrm{~kb}$ )

\section{Abbreviations}

PLS: Phosphoglycerolipids; SLs: Sphingolipids; IysoPC: Lysophosphatidylcholine; LC-MS: Liquid chromatography-mass spectrometry; PUFA: Poly-unsaturated fatty acid; ChE: Cholesteryl ester; Sul: Sulfatide; PE: Phosphatidylethanolamine; IS: Internal standard; NLs: Neutral lipids; TOF: Time-of flight:

PC: Phosphatidylcholine; PI: Phosphatidylinositol; ePC: Ether-type phosphatidylcholine; ePE: Ether-type phosphatidylethanolamine.

\section{Competing interests}

The authors declare that they have no competing interests.

\section{Authors' contributions}

Conceived and designed the experiments: KS, YK KM and YS. Performed the experiments and analysis: MI, KS and MU. Contributed reagents/materials/ analysis tools: KM and YS. Contributed to the writing of the manuscript: MI, KS and YS. All authors read and approved the final manuscript.

\section{Acknowledgements}

The authors are grateful to Katsuko Toyoshima and Mayumi Murayama for the experimental assistance, and Yuya Senoo and Kazuhisa Takemoto for assistance in data analysis. This work was financially supported by the Health Labour Sciences Research Grants (Grant number H24-Iyaku-Shitei-028 and H26-Soyaku-Ippan-008), Ministry of Health, Labour and Welfare (Tokyo, Japan), and by the Advanced Research for Products Mining Program (Grant number 10-45), National Institute of Biomedical Innovation of Japan (Osaka, Japan).

\section{Author details}

'Division of Medicinal Safety Science, National Institute of Health Sciences, 1-18-1 Kamiyoga, Setagaya, Tokyo 158-8501, Japan. ${ }^{2}$ Clinical Research Center, Kitasato University School of Medicine, 1-15-1 Kitasato, Minami, Sagamihara, Kanagawa 252-0374, Japan.

Received: 25 March 2015 Accepted: 24 August 2015

Published online: 10 September 2015

\section{References}

1. Fadok VA, Bratton DL, Rose DM, Pearson A, Ezekewitz RA, Henson PM. A receptor for phosphatidylserine-specific clearance of apoptotic cells. Nature. 2000;405:85-90.

2. Ichimura Y, Kirisako T, Takao T, Satomi Y, Shimonishi Y, Ishihara N, et al. A ubiquitin-like system mediates protein lipidation. Nature. 2000;408:488-92.

3. Kotronen A, Seppänen-Laakso T, Westerbacka J, Kiviluoto T, Arola J, Ruskeepää AL, et al. Comparison of lipid and fatty acid composition of the liver, subcutaneous and intra-abdominal adipose tissue, and serum. Obesity. 2010;18:937-44.

4. Han X, Gross RW. Shotgun lipidomics: electrospray ionization mass spectrometric analysis and quantitation of cellular lipidomes directly from crude extracts of biological samples. Mass Spectrom Rev. 2005;24:367-412.

5. Taguchi R, Nishijima M, Shimizu T. Basic analytical systems for lipidomics by mass spectrometry in Japan. Methods Enzymol. 2007;432:185-211.

6. Vouk K, Hevir N, Ribić-Pucelj M, Haarpaintner G, Scherb H, Osredkar J, et al. Discovery of phosphatidylcholines and sphingomyelins as biomarkers for ovarian endometriosis. Hum Reprod. 2012;27:2955-65.

7. Wang-Sattler R, Yu Z, Herder C, Messias AC, Floegel A, He Y, et al. Novel biomarkers for pre-diabetes identified by metabolomics. Mol Syst Biol. 2012;8:615.

8. Mapstone M, Cheema AK, Fiandaca MS, Zhong X, Mhyre TR, MacArthur LH, et al. Plasma phospholipids identify antecedent memory impairment in older adults. Nat Med. 2014:20:415-8.

9. Cheng J, Ma X, Krausz KW, Idle JR, Gonzalez FJ. Rifampicin-activated human pregnane $X$ receptor and CYP3A4 induction enhance acetaminopheninduced toxicity. Drug Metab Dispos. 2009;37:1611-21.

10. Liu JY, Li N, Yang J, Li N, Qiu H, Ai D, et al. Metabolic profiling of murine plasma reveals an unexpected biomarker in rofecoxib-mediated cardiovascular events. Proc Natl Acad Sci U S A. 2010;107:17017-22.

11. Kwan HY, Hu YM, Chan CL, Cao HH, Cheng CY, Pan SY, et al. Lipidomics identification of metabolic biomarkers in chemically induced hypertriglyceridemic mice. J Proteome Res. 2013;12:1387-98.

12. Saito K, Maekawa K, Ishikawa M, Senoo Y, Urata M, Murayama M, et al. Glucosylceramide and lysophosphatidylcholines as potential blood biomarkers for drug-induced hepatic phospholipidosis. Toxicol Sci. 2014;141:377-86.

13. Fox TE, Bewley MC, Unrath KA, Pedersen MM, Anderson RE, Jung DY, et al. Circulating sphingolipid biomarkers in models of type 1 diabetes. J Lipid Res. 2011;52:509-17.

14. Ishikawa M, Tajima Y, Murayama M, Senoo Y, Maekawa K, Saito Y. Plasma and serum from nonfasting men and women differ in their lipidomic profiles. Biol Pharm Bull. 2013;36:682-5.

15. Ishikawa M, Maekawa K, Saito K, Senoo Y, Urata M, Murayama M, et al. Plasma and serum lipidomics of healthy white adults shows characteristic profiles by subjects' gender and age. PLoS ONE. 2014;9:e91806.

16. Renner S, Römisch-Margl W, Prehn C, Krebs S, Adamski J, Göke B, et al. Changing metabolic signatures of amino acids and lipids during the prediabetic period in a pig model with impaired incretin function and reduced $\beta$-cell mass. Diabetes. 2012;61:2166-75.

17. Han X, Fagan AM, Cheng H, Morris JC, Xiong C, Holtzman DM. Cerebrospinal fluid sulfatide is decreased in subjects with incipient dementia. Ann Neurol. 2003;54:115-9.

18. Moyano AL, Pituch K, Li G, van Breemen R, Mansson JE, Givogri Ml. Levels of plasma sulfatides C18:0 and C24:1 correlate with disease status in relapsing-remitting multiple sclerosis. J Neurochem. 2013;127:600-4. 